\title{
STABILITY OF ASYMMETRIC GRAIN BOUNDARIES IN GRAPHENE
}

\author{
J. P. Mendez ${ }^{1}$, F. Arca ${ }^{2}$ and M. P. Ariza ${ }^{2}$ \\ ${ }^{1}$ Division of Engineering and Applied Science, California Institute of Technology, Pasadena, CA \\ 91125, USA \\ e-mail: jmendezg@caltech.edu \\ ${ }^{2}$ Escuela Técnica Superior de Ingeniería, Universidad de Sevilla, Camino de los descubrimientos, s.n., \\ 41092 Sevilla, Spain \\ e-mail: $\{$ farca,mpariza $\} @$ us.es
}

Keywords: Graphene, Asymmetric grain boundaries, Thermal stability.

\begin{abstract}
In the field of electronics, due to its excellent mechanical and electrical properties, graphene has become the most promising material for the production of next generation thin and flexible graphene-based electronic components. In this work, we present an assessment of the thermal stability and dynamics of asymmetric grain boundaries in graphene for different misorientation angles at finite temperature and up to extremely high temperatures. In particular, we have focused on configurations with misorientation angle of $16.1^{\circ}, 30^{\circ}$ and $38.2^{\circ}$. In contrast to pristine defect-free graphene, which has no band-gap and therefore is of limited use for semiconductor-based electronics, it has been shown theoretically that line defects in graphene might insert transport gaps, opening up the possibility of device applications based on the structural engineering of graphene boundaries.
\end{abstract}




\section{INTRODUCTION}

Graphene, owing to its extraordinary properties $[1,2]$, can be used to a wide range of applications: aerospace, where carbon fibers that are used to reinforce composite materials can be replaced by graphene and, as a result, the weight of aircraft might be reduced considerably as well as the electrical conductivity will be enhanced for lightning strikes during flights; military industry, where graphene can be used as substitution of Kevlar in bulletproof vest [3]; bioengineering, where graphene can be used to fabricate bioelectric sensors $[4,5]$; or in the field of electronics, where graphene has become the most promising material for the production of thin and flexible electronic components of next generation $[6,7]$.

However, the use of graphene in semiconductor-based devices is limited due to the fact that free-defect graphene lacks band gap, which is crucial for the correct operation of these devices. In order to achieve an electrical function and due to the presence of band gap, semiconductors exhibit the ability to switch currents on and off as desired. Many attempts have been carried out in order to introduce this desired band gap, e.g., by doping graphene with boron and nitrogen [8] or by engineering defects such as vacancies, Stone-Wales and dislocations [9], and grain boundaries (GBs) [10]. It has been proved, either experimentally or theoretically, that the presence of point defects modify locally the electronic properties [11, 12], but do not endow the layer with a band gap wide enough for such applications. Nevertheless, it has been seen that the presence of grain boundaries alters the electronic properties, and more specifically, some configurations might introduce a transport gap across them that would block the charge carriers in a range of energy. As a consequence, due to the current technology advances, the design of suited GB configurations opens a new potential via to tailor a tunable transport gap in graphene and, therefore, to use graphene as the base material for the manufacturing of semiconductor-based devices, e.g., nanotransistors [13].

With the main target of tuning the carrier transport in graphene, we will carry out an appraisal of the stability and dynamics of asymmetric GBs that could appear in graphene lattice. Additionally, owing to the fact that temperature is always present during the performance of electronic devices, the thermal stability of these GBs will play an important role when designing electronic nanocomponents. Therefore, in this work we will also conduct an analysis of the thermal stability of the predicted GB configurations during a long period of time and up to extremely high temperatures. To this end, we will proceed by applying the same methodology followed by the authors for the thermal characterization of dislocations [14] and symmetric grain boundaries [10] in graphene.

\section{ASYMMETRIC GRAIN BOUNDARIES IN GRAPHENE}

The simulation model used for studying the stability of GBs is depicted in Fig. 1. This model consists of two grains (A and B) with different crystallographic orientations $\left(\theta_{1}\right.$ and $\left.\theta_{2}\right)$ and periodic boundary conditions applied in both directions ( $\mathrm{X}$ and $\mathrm{Y}$ axis). The boundary effects are an obstacle that one has to tackle in this kind of simulations and usually emerge when the cell size is small. Owing to avoid these effects, the simulation cell has to be as large as possible, therefore, the cell size is of the order of thousands of atoms $\left(\sim 10^{3}\right)$ in this study.

The misorientation angle, $\theta$, is defined as $\theta=\theta_{1}+\theta_{2}$, where $\theta_{1}$ and $\theta_{2}$ are the orientation angles of grain $\mathrm{A}$ and $\mathrm{B}$ with respect to the horizontal axis $X$, respectively. Another interesting parameter to be considered here is the inclination angle of the boundary line, $\phi$, that is defined as $\phi=\left|\theta_{1}-\theta_{2}\right|$. On the other hand, inasmuch as graphene crystallographic structure possesses two high symmetry directions, i.e., the zig-zag and armchair directions (see Fig. 2), we fix the 


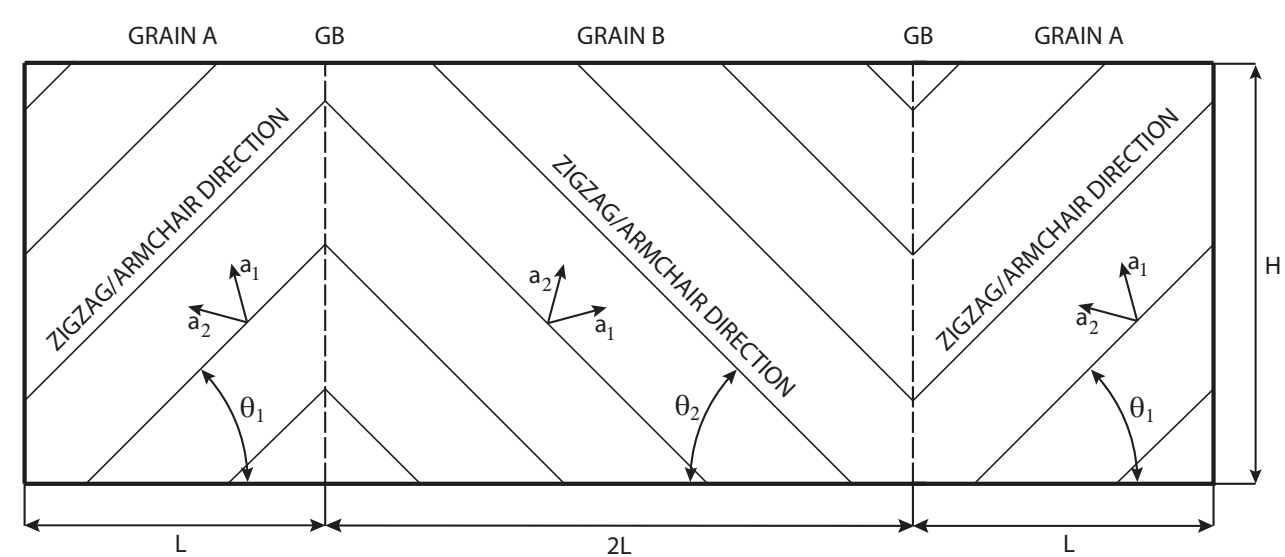

Figure 1: Grain boundary model.

zig-zag direction when the orientation angle of grain $\theta_{i}(i=1,2)$ is equal to $0^{\circ}$ and therefore the armchair direction is reached when the orientation angle $\theta_{i}(i=1,2)$ is equal to $30^{\circ}$.

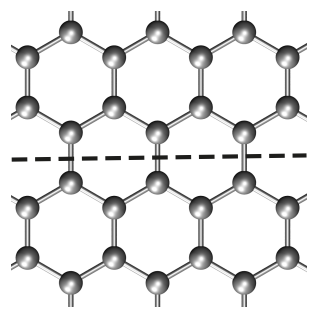

(a)

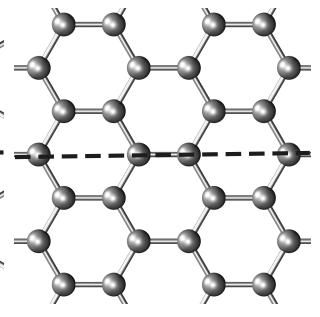

(b)

Figure 2: High symmetry directions of graphene: (a) zig-zag direction and (b) armchair direction.

Regarding grain boundary configurations, we can distinguish two types: symmetric and asymmetric. When $\theta_{1}=\theta_{2}$, GBs are symmetric and the inclination angle is $\phi=0$, whereas GBs are asymmetric when $\theta_{1} \neq \theta_{2}$ and, therefore, $\phi=\theta_{1}-\theta_{2} \neq 0$. For the purpose of evaluating all possible GB configurations, it is only necessary to cover the range of $\theta_{1}$ and $\theta_{2}$ that varies between $0^{\circ}$ and $30^{\circ}$. Therefore, symmetric configurations posses one degree of free$\operatorname{dom} \theta\left(\theta_{1}\right.$ or $\left.\theta_{2}\right)$ and have to be defined by the misorientation angle, and asymmetric GBs have two degrees of freedom $\left(\theta_{1}\right.$ and $\left.\theta_{2}\right)$ and can be denoted by $\left(\theta_{1} \mid \theta_{2}\right)$. Additionally, as GBs are traditionally modelled as a periodic arrangement of dislocations [15], they can be described by $\left(n_{A}, m_{A}\right) \mid\left(n_{B}, m_{B}\right)$, where $\left(n_{i}, m_{i}\right) i=A, B$ represents the periodic translations vector of grains $\mathrm{A}$ and $\mathrm{B}$ along GB and referred to their respective basis set $\left(a_{1}, a_{2}\right)$. In particular, $n_{A}=n_{B}$ and $m_{A}=m_{B}$ for symmetric configurations. Finally, the misorientation angle can be calculated in a straightforward manner through

$$
\theta=\arctan \left(\left(n_{A}-m_{A}\right) / \sqrt{3} /\left(m_{A}+n_{A}\right)\right)+\arctan \left(\left(m_{B}-n_{B}\right) / \sqrt{3} /\left(m_{B}+n_{B}\right)\right)
$$

\subsection{Structures and energies}

With the main purpose of finding misorientation angles corresponding to stable configurations at $0 \mathrm{~K}$, we have simulated an endless number of configurations with different angles by means of molecular dynamics, covering a wide range of possibilities. Owing to the fact that $\theta_{1} \neq \theta_{2}$ for asymmetric GBs, the characterization of these GBs is more challenging than for symmetric configurations [10]. To accomplish this, we have resorted the widely used molecular 


\begin{tabular}{ccc}
\hline $\begin{array}{c}\text { Misorientation angle }(\theta) \\
\theta\left(\theta_{A} \mid \theta_{B}\right)\end{array}$ & $\begin{array}{c}\text { GB configuration } \\
\left(n_{A}, m_{A}\right) \mid\left(n_{B}, m_{B}\right)\end{array}$ & $\begin{array}{c}\text { Energy } \\
(\mathrm{eV} / \AA)\end{array}$ \\
\hline \hline $16.1^{\circ}\left(16.1^{\circ} \mid 0^{\circ}\right)$ & $(3,1) \mid(2,2)$ & 0.932 \\
$16.1^{\circ}\left(0^{\circ} \mid 16.1^{\circ}\right)$ & $(2,2) \mid(1,3)$ & 0.323 \\
$30^{\circ}\left(30^{\circ} \mid 0^{\circ}\right)$ & $(5,0) \mid(3,3)$ & 0.361 \\
$30^{\circ}\left(0^{\circ} \mid 30^{\circ}\right)$ & $(3,3) \mid(0,5)$ & 1.241 \\
$38.2^{\circ}\left(8.21^{\circ} \mid 30^{\circ}\right)$ & $(5,3) \mid(0,7)$ & 0.394 \\
\hline
\end{tabular}

Table 1: Energies for different asymmetric grain boundaries in graphene.

dynamics code LAMMPS (Large-scale Atomic/Molecular Massively Parallel Simulator) and the AIREBO [16] and ReaxFF potentials [17] that are implemented in LAMMPS code. The strategy proceeded to find these configurations is as follows: first, employing the ReaxFF potential and NVE ensemble, we grow two grains with different misorientation angles and force them to interact and form an interface between both grains. It should be noted that sometimes it is necessary to remove atoms along the interface in order to achieve the desired grain boundary structure; and second, we stabilize the GB structure using the AIREBO potential and the Nose-Hoover thermostat (NVT ensemble) at very low constant temperature $(\sim 0 \mathrm{~K})$.

In our simulations, we have adopted that GBs are constituted by periodic arrays of dislocations. This periodicity can be defined by two periodic translation vectors, $\left(n_{A}, m_{A}\right)$ and $\left(n_{B}, m_{B}\right)$, belonging to grains $\mathrm{A}$ and $\mathrm{B}$, respectively, and parallel to the GB (see Fig. 1). Unlike symmetric configurations, these translation vectors might differ slightly in magnitude for some asymmetric configurations, $d_{A} \neq d_{B}\left(d_{A}=2.46\left(n_{A} \cos \left(30-\theta_{1}\right)+m_{A} \cos \left(30+\theta_{1}\right)\right)\right.$ and $\left.d_{B}=2.46\left(n_{B} \cos \left(30+\theta_{2}\right)+m_{B} \cos \left(30-\theta_{2}\right)\right)\right)$. However, in order to form stable asymmetric $\mathrm{GB}$, the mismatch between both domains at the GB must be quite small and its stored energy must be reasonably low. In order to evaluate the energies and structures of these asymmetric configurations, the height of the computational cell could be considered proportional to $d_{A}$ or to $d_{B}$. In our model, we take the height of the computational cell $H$ proportional to $d_{A}$.

In Fig. 3, we present the obtained asymmetric configurations in our calculations, and the corresponding energies for all possible cases and the misorientation angles of $16.1^{\circ}, 30^{\circ}$ and $38.2^{\circ}$ are tabulated in Table 2.1. First, we have found that asymmetric configurations do not follow the same pattern as symmetric configurations, and a large number of dislocation types and distribution along the grain boundary can be found. For instance, the GB structures with misorientation angles of $\theta=30^{\circ}$ and $\theta=38.2^{\circ}$ are constructed by $(1,0)+(0,1)$ and $(1,0)$ dislocations, whereas $(1,0)$ dislocations are arranged along an interface of two grains with a misorientation angle of $16.1^{\circ}$ (see Figs. 3 and 4). These results have also been corroborated by previous studies $[18,19]$. Second, we have found that our low energy configurations correspond to those with $d_{A}<d_{B}$. Third, for these low energy configurations, our simulations show the existence of out-of-plane displacement, about $0.5 \AA$.

Finally, these results have been also corroborated experimentally, [20] observed GBs with misorientation angles of $\sim 16^{\circ}$ and $\sim 29^{\circ}$ which can be related to the angles of $16.1^{\circ}$ and $30^{\circ}$ in our calculations, respectively.

\section{Thermal stability}

Afterwards, we have analysed the mechanical behaviour and thermal stability at finite temperature of these GB structures up to extremely high temperatures. For this purpose, we have relaxed each configuration during a long period of time, up to $10.000 \mathrm{ps}$, and at fixed constant 


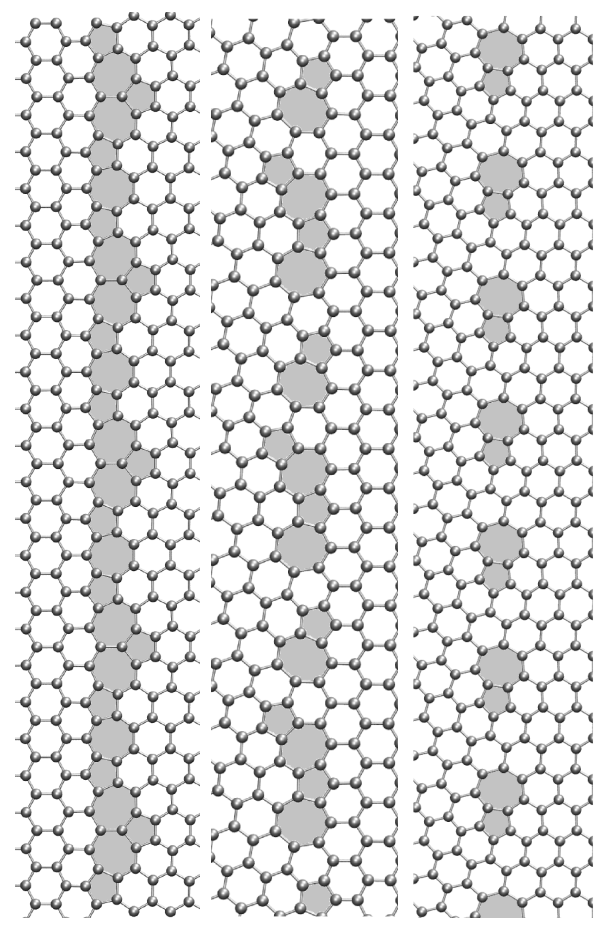

(a)

(b)

(c)

Figure 3: Observed structures of GB in graphene lattice (a) $30^{\circ} \mid 0^{\circ}\left(\theta=30^{\circ}\right)$ or $(5,0) \mid(3,3)$ (b) $8.21^{\circ} \mid 30^{\circ}\left(\theta=38.21^{\circ}\right)$ or $(5,3) \mid(0,7)$ and (c) $16.1^{\circ} \mid 0^{\circ}\left(\theta=16.1^{\circ}\right)$ or $(3,1) \mid(2,2)$.

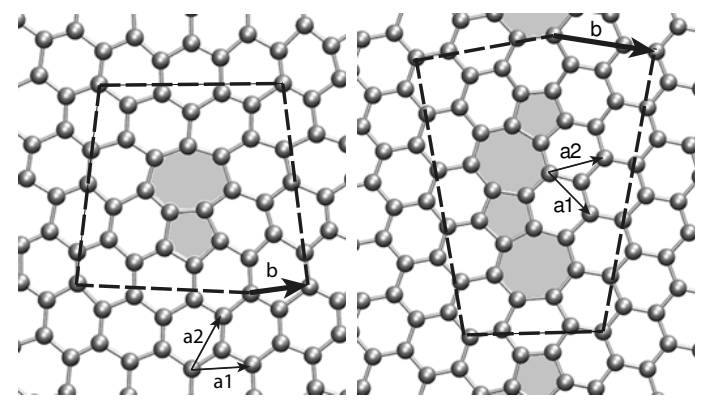

(a)

(b)

Figure 4: Dislocation type (a) $(1,0)$ and (b) $(1,0)+(0,1)$.

temperature, covering a wide range of temperatures from $0 \mathrm{~K}$ to temperatures close to the melting point of graphene [21, 22, 23]. Similarly, to carry out the thermal stability analysis, we have employed the LAMMPS code, the full AIREBO potential and the NVT ensemble ( $\Delta t=0.1 \mathrm{fs}$ ). As main outcomes of our simulations, we state the following conclusions:

1. Asymmetric configurations remain stable up to very high temperature, around $3000 \mathrm{~K}$. As we can observe from Fig. 5a for a misorientation angle of $38.2^{\circ}$, dislocations type $(1,0)$ and $(1,0)+(0,1)$ which form the grain boundary are unaffected by the temperature.

2. At temperature exceeding $3000 \mathrm{~K}$, it is observed transformations, motions and slip-up of dislocations. For instance, as we can see in the rectangles marked in Figs. 5b and c, a $(1,0)+(0,1)$ dislocation segregate into two dislocations, and the resulting dislocations move and one of them joins to a $(1,0)$ dislocation to form new dislocation. Additionally, we show in Fig. 6 as an example the glide motion of a $(1,0)$ dislocation. 


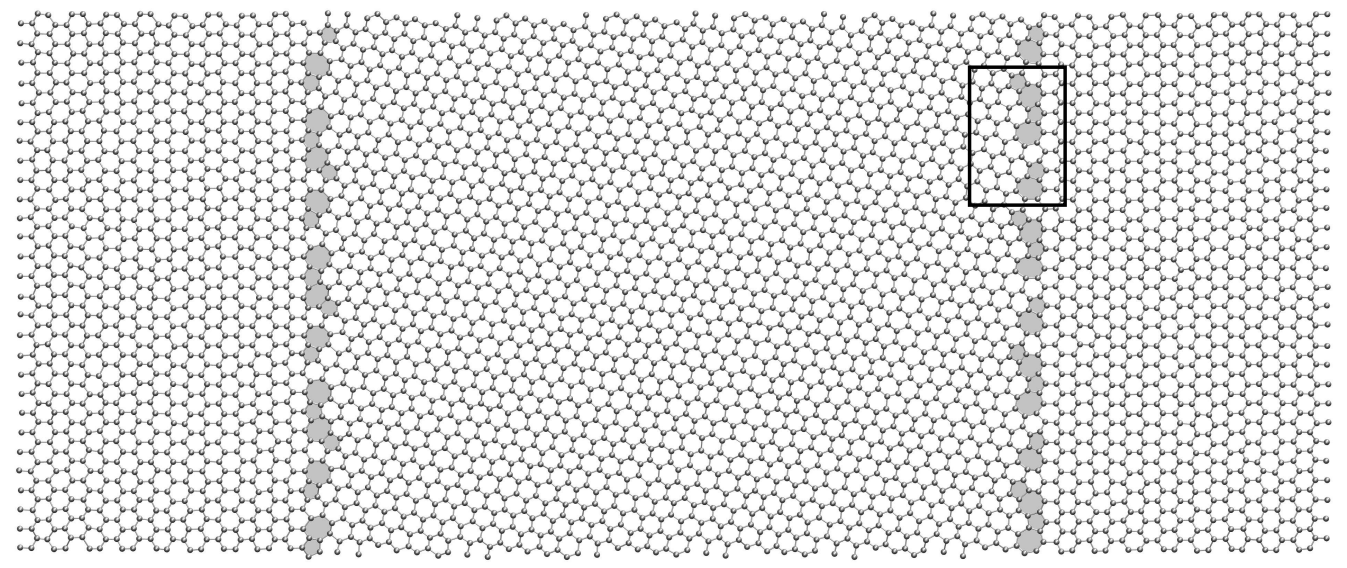

(a)

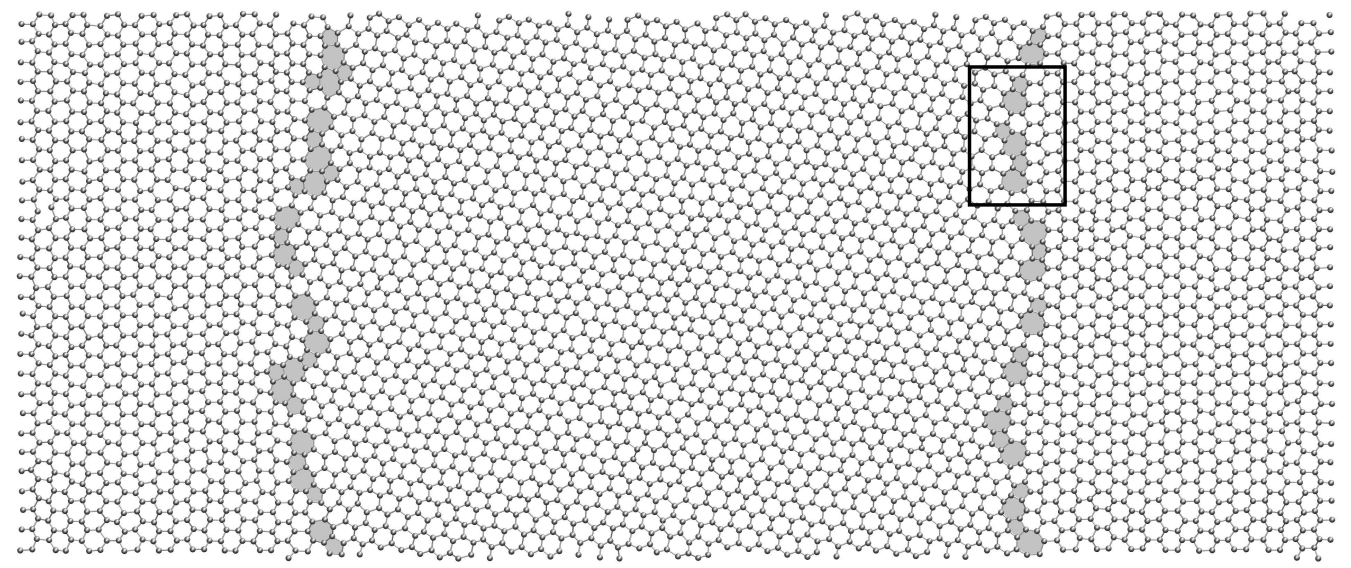

(b)

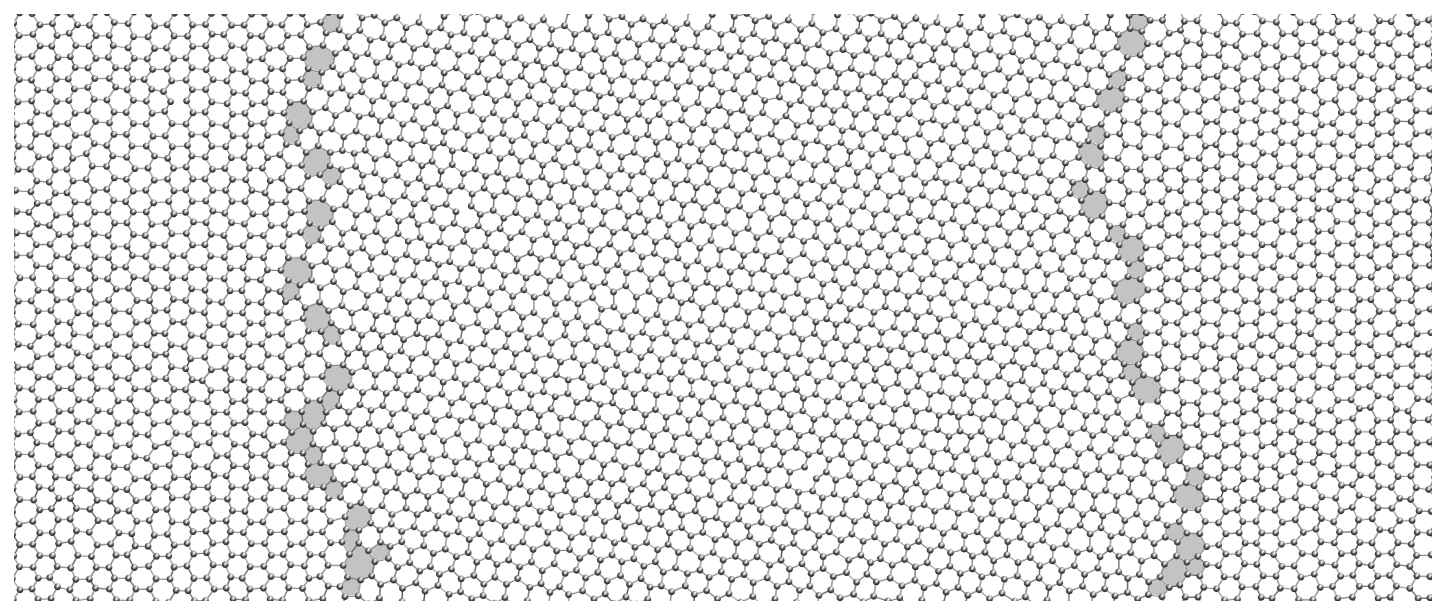

(c)

Figure 5: Observed asymmetric grain boundary structures for a misorientation angle of $38.2^{\circ}$ at different temperatures (a) $\mathrm{T} \leq 3000 \mathrm{~K}$, (b) $\mathrm{T}=3500 \mathrm{~K}$, and (c) $\mathrm{T}=4000 \mathrm{~K}$. 


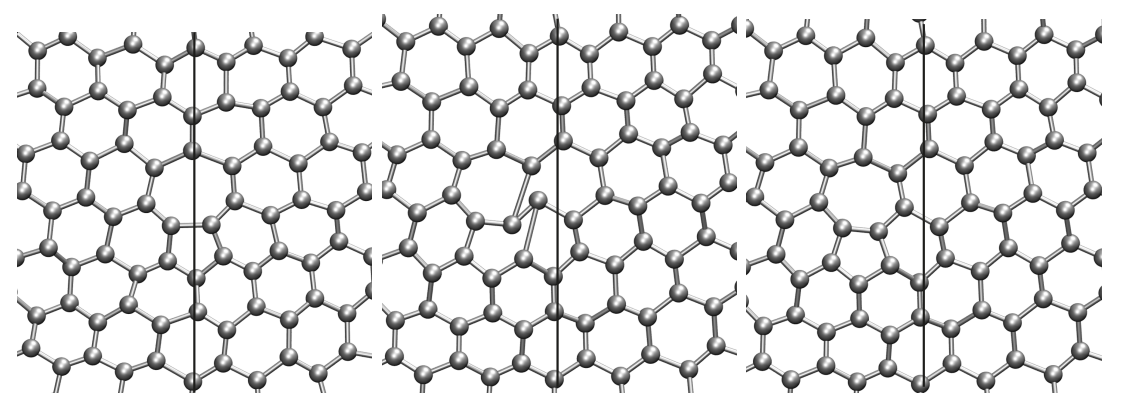

(a)

(b)

(c)

Figure 6: Details of the glide motion of $(1,0)$ dislocations showing at temperatures above $2000 \mathrm{~K}$.

3. Above 4000K, asymmetric GBs collapse. Similar to symmetric configurations, the presence of GBs accelerates the melting process and decreases the melting temperature. Therefore, these defects act as a trigger for melting.

\section{CONCLUSIONS}

In the present work, we have analysed the dynamics and the thermal stability of asymmetric GBs for different misorientation angles. First, we have obtained atomic configurations corresponding to stable GBs using the LAMMPS code and the AIREBO and ReaxFF potentials. In particular, we have obtained that the misorientation angles $16.1^{\circ}, 30^{\circ}$ and $38.2^{\circ}$ are stable in graphene. Afterwards, we have relaxed these configurations at finite temperature up to very high temperature, close to the melting temperature for graphene. As results, we have reported a high stability against annihilation up to extreme temperatures, about $3000 \mathrm{~K}$, high mobility and transformation of dislocations at higher temperatures and the melting of grain boundaries at temperarutes above $4000 \mathrm{~K}$.

\section{REFERENCES}

[1] A. H. Castro Neto, F. Guinea, N. M. R. Peres, K. S. Novoselov, and A. K. Geim. The electronic properties of graphene. Rev. Mod. Phys., 81:109-162, 2009.

[2] I. W. Frank, D. M. Tanenbaum, A. M. Van der Zande, and P. L. McEuen. Mechanical properties of suspended graphene sheets. Journal of Vacuum Science \& Technology B, 25(6):2558-2561, 2007.

[3] Jae-Hwang Lee, Phillip E. Loya, Jun Lou, and Edwin L. Thomas. Dynamic mechanical behavior of multilayer graphene via supersonic projectile penetration. Science, 346(6213):1092-1096, 2014.

[4] Y. Shao, J. Wang, H. Wu, J. Liu, I.A. Aksay, and Y. Lin. Graphene based electrochemical sensors and biosensors: A review. Electroanalysis, 22(10):1027-1036, 2010.

[5] Z. Tang, H. Wu, J.R. Cort, G.W. Buchko, Y. Zhang, Y. Shao, I.A. Aksay, J. Liu, and Y. Lin. Constraint of dna on functionalized graphene improves its biostability and specificity. Small, 6(11):1205-1209, 2010. 
[6] S. Bae, H. Kim, Y. Lee, X. Xu, J.S. Park, Y. Zheng, J. Balakrishnan, T. Lei, H. R. Kim, Y. I. Song, Y.J. Kim, K.S. Kim, B. zyilmaz, J.H. Ahn, B. H. Hong, and S. Iijima. Roll-to-roll production of 30-inch graphene films for transparent electrodes. Nature Nanotechnology, 5(8):574-578, 2010.

[7] Y.U. Jung, K.W. Park, S.T. Hur, S.W. Choi, and S.J. Kang. High-transmittance liquidcrystal displays using graphene conducting layers. Liquid Crystals, 0(0):1-5, 2013.

[8] Pooja Rani and VK Jindal. Designing band gap of graphene by $\mathrm{b}$ and $\mathrm{n}$ dopant atoms. RSC Advances, 3(3):802-812, 2013.

[9] M.P. Ariza and J.P. Mendez. Stability of discrete topological defects in graphene. Journal of Mechanics of Materials and Structures, 10(2):239-253, 2015.

[10] J.P. Mendez, F. Macias, and M.P. Ariza. Thermal stability of tilt grain boundaries in graphene. Applied Mechanics and Materials, 481:129-132, 2014.

[11] Sharmila N. Shirodkar and Umesh V. Waghmare. Electronic and vibrational signatures of stone-wales defects in graphene: First-principles analysis. Phys. Rev. B, 86:165401, Oct 2012.

[12] A. Lherbier, S.M.M. Dubois, X. Declerck, Y.M. Niquet, S. Roche, and J.C. Charlier. Transport properties of graphene containing structural defects. Physical Review B, 86:075402, Aug 2012.

[13] B. Standley, W. Bao, J. Zhang, H.and Bruck, C. N. Lau, and M. Bockrath. Graphene-based atomic-scale switches. Nano Letters, 8(10):3345-3349, 2008.

[14] M.P. Ariza, M. Ortiz, and R. Serrano. Long-term dynamic stability of discrete dislocations in graphene at finite temperature. International Journal of Fracture, 166(1-2):215-223, 2010.

[15] W. T. Read and W. Shockley. Dislocation models of crystal grain boundaries. Phys. Rev., 78:275-289, May 1950.

[16] S. J. Stuart, A. B. Tutein, and J. A. Harrison. A reactive potential for hydrocarbons with intermolecular interactions. J. Chem. Phys., 112(14):6472-6486, 2000.

[17] Adri C. T. van Duin, Siddharth Dasgupta, Francois Lorant, and William A. Goddard. Reaxff: A reactive force field for hydrocarbons. The Journal of Physical Chemistry A, 105(41):9396-9409, 2001.

[18] Jihoon Han, Seunghwa Ryu, Dongwoo Sohn, and Seyoung Im. Mechanical strength characteristics of asymmetric tilt grain boundaries in graphene. Carbon, 68(0):250 - 257, 2014.

[19] Xiuyun Zhang, Ziwei Xu, Qinghong Yuan, John Xin, and Feng Ding. The favourable large misorientation angle grain boundaries in graphene. Nanoscale, 7(47):20082-20088, 2015 . 
[20] Jinho An, Edgar Voelkl, Ji Won Suk, Xuesong Li, Carl W. Magnuson, Lianfeng Fu, Peter Tiemeijer, Maarten Bischoff, Bert Freitag, Elmira Popova, and Rodney S. Ruoff. Domain (grain) boundaries and evidence of twinlike structures in chemically vapor deposited grown graphene. ACS Nano, 5(4):2433-2439, 2011. PMID: 21361332.

[21] K V Zakharchenko, Annalisa Fasolino, J H Los, and M I Katsnelson. Melting of graphene: from two to one dimension. Journal of Physics: Condensed Matter, 23(20):202202, 2011.

[22] Y Kowaki, A Harada, F Shimojo, and K Hoshino. Radius dependence of the melting temperature of single-walled carbon nanotubes: molecular-dynamics simulations. Journal of Physics: Condensed Matter, 19(43):436224, 2007.

[23] Seong Gon Kim and David Tománek. Melting the fullerenes: A molecular dynamics study. Phys. Rev. Lett., 72:2418-2421, Apr 1994. 